\title{
Uniquely $C_{4}$-Saturated Graphs*
}

\author{
Joshua Cooper $\ddagger \quad$ John Lenz; ${ }^{\ddagger}$ Timothy D. LeSaulnier; \\ Paul S. Wenger; Douglas B. West ${ }^{\ddagger}$
}

January 26, 2010

\begin{abstract}
For a fixed graph $H$, a graph $G$ is uniquely $H$-saturated if $G$ does not contain $H$, but the addition of any edge from $\bar{G}$ to $G$ completes exactly one copy of $H$. Using a combination of algebraic methods and counting arguments, we determine all the uniquely $C_{4}$-saturated graphs; there are only ten of them.
\end{abstract}

\section{Introduction}

For a fixed graph $H$, a graph $G$ is $H$-saturated if $G$ does not contain $H$ but joining any nonadjacent vertices produces a graph that does contain $H$. Let $P_{n}, C_{n}, K_{n}$ denote the path, cycle, and complete graph with $n$ vertices, respectively. The study of $H$-saturated graphs began when Turán [5] determined the $n$-vertex $K_{r}$-saturated graphs with the most edges. In the opposite direction, Erdös, Hajnal, and Moon [1] determined the $n$-vertex $K_{r^{-}}$ saturated graphs with the fewest edges. A survey of results and problems about the smallest $n$-vertex $H$-saturated graphs appears in [4].

A graph $G$ is uniquely $H$-saturated if $G$ is $H$-saturated and the addition of any edge joining nonadjacent vertices completes exactly one copy of $H$. The graphs found in [1] are uniquely $K_{r}$-saturated. For example, consider $H=C_{3}$. Every $C_{3}$-saturated graph has diameter at

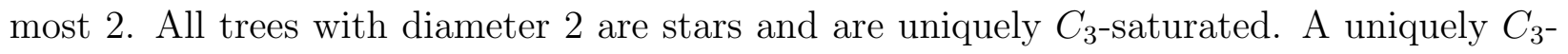
saturated graph $G$ cannot contain a 3-cycle or a 4-cycle, so such a graph that is not a tree

${ }^{*}$ Research of all authors partially supported by NSF grant DMS 08-38434 "EMSW21-MCTP: Research Experience for Graduate Students."

${ }^{\dagger}$ Mathematics Dept., Univ. of South Carolina, Columbia, SC; cooper@math.sc.edu.

${ }_{\ddagger}^{\ddagger}$ Mathematics Dept., Univ. of Illinois, Urbana, IL; email addresses jlenz2@illinois.edu, tlesaul2@illinois.edu, pwenger2@illinois.edu, west@math.uiuc.edu.

${ }^{\S}$ Research supported by NSA grant H98230-09-1-0363. 
has girth 5 . Every graph with girth 5 and diameter 2 is uniquely $C_{3}$-saturated. The graphs with diameter $d$ and girth $2 d+1$ are the Moore graphs. Hoffman and Singleton [2] proved that besides odd cycles there are only finitely many Moore graphs, all having diameter 2. Thus, except for stars, there are finitely many uniquely $C_{3}$-saturated graphs.

Ollmann [3] determined the $C_{4}$-saturated $n$-vertex graphs with the fewest edges, but few of these are uniquely $C_{4}$-saturated. An exception is the triangle $K_{3}$; whenever $n<|V(H)|$, vacuously $K_{n}$ is uniquely $H$-saturated. In this paper we determine all the uniquely $C_{4^{-}}$ saturated graphs.

Theorem 1. There are precisely ten uniquely $C_{4}$-saturated graphs.

In the list, the only example with girth 5 is the 5 -cycle. The others are small trees or contain triangles; all have at most nine vertices.

The sense in which uniquely $C_{k}$-saturated graphs can be viewed as generalizing the Moore graphs of diameter 2 is reflected in our proof. The structure and techniques of the paper are very similar to the eigenvalue approach used to prove both the Hoffman-Singleton result on Moore graphs and the "Friendship Theorem", which states that a graph in which any two distinct vertices have exactly one common neighbor has a vertex adjacent to all others (see Wilf [7]). Structural arguments are used to show that under certain conditions the graphs in question are regular. Counting of walks then yields a polynomial equation involving the adjacency matrix, after which eigenvalue arguments exclude all but a few graphs.

The graphs that result from the Friendship Theorem consist of some number of triangles sharing a single vertex; such graphs are uniquely $C_{5}$-saturated. Thus, unlike for $C_{4}$, there are infinitely many uniquely $C_{5}$-saturated graphs. Wenger [6] has shown that except for small complete graphs, the "friendship graphs" are the only uniquely $C_{5}$-saturated graphs.

\section{Structural Properties}

Our graphs have no loops or multi-edges. A $k$-cycle is a cycle with $k$ vertices, and we define a $k$-path to be a $k$-vertex path. A path with endpoints $x$ and $y$ is an $x, y$-path. For a vertex $v$ in a graph $G$, the neighborhood $N(v)$ is $\{u \in V(G): u v \in E(G)\}$. The kth neighborhood $N^{k}(v)$ is $\{u \in V(G): d(u, v)=k\}$, where the distance $d(u, v)$ is the minimum length of a $u, v$-path. The diameter of a graph is the maximum distance between vertices. The degree $d(v)$ of a vertex $v$ in a graph $G$ is the number of incident edges.

We begin with basic observations about the structure of uniquely $C_{4}$-saturated graphs. 
Lemma 2. The following properties hold for every uniquely $C_{4}$-saturated graph $G$.

(a) $G$ is connected and has diameter at most 3.

(b) Any two nonadjacent vertices in $G$ are the endpoints of exactly one 4-path.

(c) $G$ contains no 6-cycle and no two triangles sharing a vertex.

Proof. If $x$ and $y$ are nonadjacent vertices in $G$, then the edge $x y$ completes a 4-cycle. Thus $G$ contains an $x, y$-path of length 3 . Since $G$ is uniquely $C_{4}$-saturated, $x$ and $y$ are the endpoints of exactly one 4-path. Opposite vertices on a 6-cycle would be the endpoints of two 4-paths if nonadjacent and would lie on a 4-cycle if adjacent. The same is true for nonadjacent vertices in the union of two triangles sharing one vertex. The union of two triangles sharing two vertices contains a 4-cycle.

Lemma 3. If $G$ is uniquely $C_{4}$-saturated and $|V(G)| \geq 3$, then $G$ has girth 3 or 5 .

Proof. If $G$ contains a triangle, then $G$ has girth 3, so we may assume that $G$ is triangle-free. Hence there are vertices $x$ and $y$ with $d(x, y)=2$; let $z$ be their unique common neighbor. By Lemma 2, there is a 4-path joining $x$ and $y$. If it contains $z$, then $G$ contains a triangle. Otherwise, $x$ and $y$ lie on a 5 -cycle. Since $G$ is $C_{4}$-free, it follows that $G$ has girth 5 .

If $G$ has maximum degree at most 1 , then $G$ is $K_{1}$ or $K_{2}$, and these are uniquely $C_{4^{-}}$ saturated. We may assume henceforth maximum degree at least 2. Lemma 3 then allows us to break the study of uniquely $C_{4}$-saturated graphs into two cases: girth 3 and girth 5 .

\section{Girth 5}

Lemma 4. If $G$ is a uniquely $C_{4}$-saturated graph with girth 5 , then $G$ is regular.

Proof. Let $u$ and $v$ be adjacent vertices, with $d(u) \leq d(v)$. Since $G$ is triangle-free, $N(v)$ is an independent set, and hence the 4-paths joining neighbors of $v$ do not contain $v$. If $d(u)<d(v)$, then by the pigeonhole principle two of the unique 4-paths from $u$ to the other $d(v)-1$ neighbors of $v$ begin along the same edge $u u^{\prime}$ incident to $u$. Each of these two paths continues along an edge to $v$ to form distinct 4-paths from $u^{\prime}$ to $v$. Since $N(v)$ is independent, $u^{\prime}$ is not adjacent to $v$, so this contradicts Lemma 2.

We conclude that adjacent vertices in $G$ have the same degree. Since $G$ is connected, it follows that $G$ is $k$-regular.

We now show that exactly one uniquely $C_{4}$-saturated graph has girth 5 . 
Theorem 5. The only uniquely $C_{4}$-saturated graph with girth 5 is $C_{5}$.

Proof. Let $G$ be a uniquely $C_{4}$-saturated $n$-vertex graph with girth 5 . By Lemma $4, G$ is regular; let $k$ be the vertex degree. Let $A$ be the adjacency matrix of $G$, let $J$ be the $n$-by-n matrix with every entry 1 , and let 1 be the $n$-vector with each coordinate 1 . If $x$ and $y$ are nonadjacent vertices of $G$, then by Lemma 2 there is one $x, y$-path of length 3 and no other walk of length 3 joining $x$ and $y$. If $x$ and $y$ are adjacent, then there are $2 k-1$ walks of length 3 joining them. If $x=y$, then no walk of length 3 joins $x$ and $y$, because $G$ is triangle-free. This yields $A^{3}=(J-A-I)+(2 k-1) A$, or $J=A^{3}-(2 k-2) A+I$.

Because $J$ is a polynomial in $A$, every eigenvector of $A$ is also an eigenvector of $J$. Since $G$ is $k$-regular, $\mathbf{1}$ is an eigenvector of $A$ with eigenvalue $k$. Also $\mathbf{1}$ is an eigenvector of $J$ with eigenvalue $n$. This yields the following count of the vertices of $G$ :

$$
n=k^{3}-(2 k-2) k+1=k^{3}-2 k^{2}+2 k+1 .
$$

We have observed that every eigenvector of $A$ is also an eigenvector of $J$. Since $J$ has rank 1, we conclude that $J x=0 x$ when $x$ is an eigenvector of $A$ other than 1 . If $\lambda$ is the corresponding eigenvalue of $A$, then $J=A^{3}-(2 k-2) A+I$ yields

$$
0=\lambda^{3}-(2 k-2) \lambda+1 .
$$

It follows that $A$ has at most three eigenvalues other than $k$.

Let $q$ denote the polynomial in (1). Being a cubic polynomial, it factors as

$$
q(\lambda)=\lambda^{3}-(2 k-2) \lambda+1=\left(\lambda-r_{1}\right)\left(\lambda-r_{2}\right)\left(\lambda-r_{3}\right) .
$$

It follows that

$$
r_{1}+r_{2}+r_{3}=0
$$

Suppose first that two of these roots have a common value, $r$. From (3), the third is $-2 r$, and we have

$$
\lambda^{3}-(2 k-2) \lambda+1=(\lambda-r)^{2}(\lambda+2 r)=\lambda^{3}-3 r^{2} \lambda+2 r^{3} .
$$

By equating coefficients, $r$ equals both $(1 / 2)^{1 / 3}$ (irrational) and $(2 k-2) / 3$ (rational). Hence $q$ has three distinct roots.

Suppose next that $q$ has a rational root. The Rational Root Theorem implies that 1 and -1 are the only possible rational roots of $q$. If -1 is a root, then $k=1$ and $G$ does not have girth 5. If 1 is a root, then $k=2$ and $G=C_{5}$. 
Hence we may assume that $q$ has three distinct irrational roots. In this case we will obtain a contradiction. Index the eigenvalues so that the multiplicities $a, b$, and $c$ of $r_{1}, r_{2}$, and $r_{3}$ (respectively) satisfy $a \leq b \leq c$. Letting $p_{A}$ be the characteristic polynomial of $A$,

$$
p_{A}(\lambda)=(\lambda-k)\left(\lambda-r_{1}\right)^{a}\left(\lambda-r_{2}\right)^{b}\left(\lambda-r_{3}\right)^{c} .
$$

Combining (2) and (4) yields

$$
p_{A}(\lambda)=(\lambda-k)\left(\lambda^{3}-(2 k-2) \lambda+1\right)^{a}\left(\lambda-r_{2}\right)^{b-a}\left(\lambda-r_{3}\right)^{c-a} .
$$

Because $A$ has integer entries, $p_{A}(\lambda) \in \mathbb{Q}[\lambda]$. By applying the division algorithm, $p=r s$ and $p, r \in \mathbb{Q}[\lambda]$ imply $s \in \mathbb{Q}[\lambda]$. Hence $\left(\lambda-r_{2}\right)^{b-a}\left(\lambda-r_{3}\right)^{c-a} \in \mathbb{Q}[\lambda]$. Since $q(\lambda)$ is a monic cubic polynomial in $\mathbb{Q}[\lambda]$ with three irrational roots, it is irreducible and is the minimal polynomial of $r_{1}, r_{2}$, and $r_{3}$ over $\mathbb{Q}$. Thus $q$ divides $\left(\lambda-r_{2}\right)^{b-a}\left(\lambda-r_{3}\right)^{c-a}$ if $c>a$. In that case, since $r_{1}$ is a root of $q$, it is also a root of $\left(\lambda-r_{2}\right)^{b-a}\left(\lambda-r_{3}\right)^{c-a}$. We conclude that $c=a$, and all three eigenvalues have the same multiplicity.

The trace of $A$ is 0 , so

$$
k+a r_{1}+a r_{2}+a r_{3}=k+a\left(r_{1}+r_{2}+r_{3}\right)=\operatorname{Tr}(A)=0
$$

Together, (3) and (5) require $k=0$. Thus $q$ cannot have three distinct irrational roots when $G$ has girth 5 .

\section{Girth 3}

We now consider uniquely $C_{4}$-saturated graphs with a triangle. The next lemma gives a structural decomposition. For a set $S \subseteq V(G)$, let $d(x, S)=\min \{d(x, v): v \in S\}$, let $N(S)=\{v \in V(G): d(v, S)=1\}$, and let $N^{k}(S)=\{v \in V(G): d(v, S)=k\}$.

Lemma 6. Let $S$ be the vertex set of a triangle in a graph $G$, with $S=\left\{v_{1}, v_{2}, v_{3}\right\}$. For $i \in\{1,2,3\}$, let $V_{i}=N\left(v_{i}\right)-S$, and let $V_{i}^{\prime}=N^{2}\left(v_{i}\right)-N(S)$. Let $R=N^{3}(S)$. If $G$ is uniquely $C_{4}$-saturated, then $G$ has the following structure:

(a) $V_{i} \cap V_{j}=\varnothing$ when $i \neq j$;

(b) each vertex in $V_{i}^{\prime}$ has exactly one neighbor in $V_{i}$;

(c) $V_{i}^{\prime} \cap V_{j}^{\prime}=\varnothing$ when $i \neq j$;

(d) no edges join $V_{i}^{\prime}$ and $V_{j}^{\prime}$ when $i \neq j$;

(e) $N(S)$ is independent;

(f) each $V_{i}^{\prime}$ induces a matching;

(g) each vertex in $R$ has exactly one neighbor in each $V_{i}^{\prime}$. 


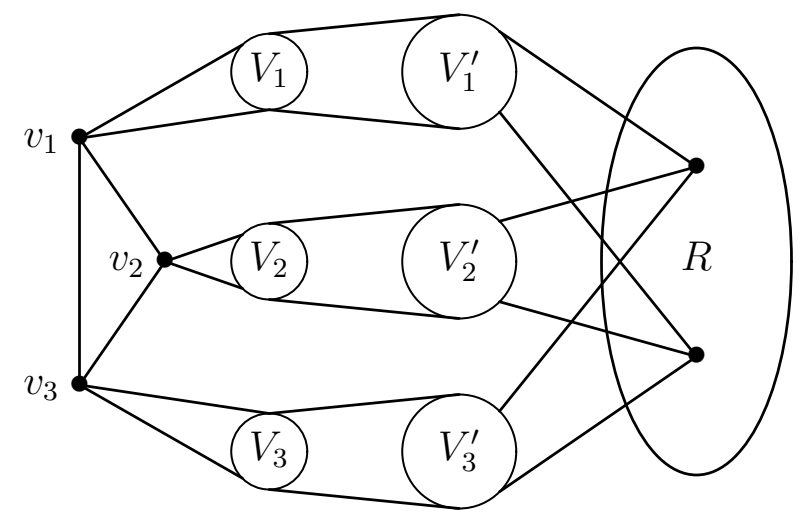

Figure 1: Structure of uniquely $C_{4}$-saturated graph with a triangle.

Proof. Since $G$ has diameter 3, we have described all of $V(G)$. Figure 1 makes it easy to see most of the conclusions. The prohibition of 4-cycles and of triangles with common vertices implies (a), (b), and (e). The prohibition of 6-cycles implies (c) and (d).

Given these results, (f) is implied by the existence of a unique 4-path joining $v_{i}$ to each vertex of $V_{i}^{\prime}$. For $(\mathrm{g})$, each vertex in $R$ is joined by a unique 4-path to each vertex in $S$; it can only reach $v_{i}$ quickly enough by moving first to a vertex of $V_{i}^{\prime}$, and uniqueness of the 4-path prohibits more than one such neighbor.

The main part of the argument is analogous to the regularity, walk-counting, and eigenvalue arguments in Lemma 4 and Theorem 5.

Theorem 7. If $G$ is a $C_{4}$-saturated graph with a triangle, then $R=\varnothing$ in the partition of $V(G)$ given in Lemma 6.

Proof. If $R \neq \varnothing$, then each set $V_{i}$ and $V_{i}^{\prime}$ in the partition is nonempty. We show first that $G$ is regular, then show that each vertex lies in one triangle, and finally count 4-paths to determine the cube of the adjacency matrix and obtain a contradiction using eigenvalues.

Consider $V_{i}^{\prime}$ and $V_{j}$ with $i \neq j$. A vertex $x$ in $V_{i}^{\prime}$ reaches each vertex of $V_{j}$ by a unique 4-path, passing through $R$ and $V_{j}^{\prime}$. By Lemma $6(\mathrm{~g})$, each vertex of $R$ has one neighbor in $V_{j}^{\prime}$, so each edge from $x$ to $R$ starts exactly one 4-path to $V_{j}$. By Lemma 6 , the other neighbors of $x$ are one each in $V_{i}$ and $V_{i}^{\prime}$, so $d(x)=\left|V_{j}\right|+2$. Since the choice of $i$ and $j$ was arbitrary, we conclude that each vertex of $N^{2}(S) \cup S$ has degree $a+2$, where $a=\left|V_{1}\right|=\left|V_{2}\right|=\left|V_{3}\right|$.

For $x \in V_{i}$ and $y \in V_{j}$ with $j \neq i$, the unique 4-path joining $x$ to any neighbor of $y$ in $V_{j}^{\prime}$ must pass through $V_{i}^{\prime}$ and $R$. By Lemma $6(\mathrm{~g})$, these paths use distinct vertices in $R$; since 
$G$ has no 6-cycle through $y$, they also use distinct vertices in $V_{i}^{\prime}$. Hence $d(x) \geq d(y)$. By symmetry, all vertices of $N(S)$ have the same degree; let this degree be $b+1$.

Consider $r \in R$. By Lemma 6(g), 4-paths from $r$ to $V_{i}$ may visit another vertex in $R$ and then reach $V_{i}$ in exactly one way, or they may go directly to $V_{i}^{\prime}$, traverse an edge within $V_{i}^{\prime}$, and continue to $V_{i}$. The total number of such paths is $[d(r)-3]+1$, and this must equal $\left|V_{i}\right|$. Hence $d(r)=a+2$. Since $\left|V_{i}\right|=a$ and $d(x)=b+1$ for $x \in V_{i}$, Lemma 6 yields $\left|V_{i}^{\prime}\right|=a b$.

Consider $x \in V_{i}^{\prime}$ and $j \neq i$. Each 4-path from $x$ to $V_{j}^{\prime}$ starts with an edge in $V_{i}^{\prime}$, ends with an edge in $V_{j}^{\prime}$, or uses two vertices in $R$. Since each vertex in $N^{2}(S)$ has a neighbors in $R$, there are $a$ paths of each of the first two types. Since each vertex of $R$ has degree $a+2$, with three neighbors in $N^{2}(S)$, there are $a(a-1)$ paths of the third type. Since these paths reach distinct vertices of $V_{j}^{\prime}$, and every vertex of $V_{j}^{\prime}$ is reached, $\left|V_{j}^{\prime}\right|=a(a+1)$.

Hence $a(a+1)=a b$, and $b=a+1$. Since every vertex of $G$ has degree $a+2$ or $b+1$, we conclude that $G$ is $k$-regular, where $k=a+2$.

We show next that every vertex of $G$ lies in a triangle. If $v$ lies in no triangle, then $N(v)$ is independent, and having unique 4-paths from $N^{2}(v)$ to $v$ forces $N^{2}(v)$ to induce a 1-regular subgraph. Since $\left|N^{2}(v)\right|=k(k-1)$, there are $\left(\begin{array}{c}k \\ 2\end{array}\right)$ edges induced by $N^{2}(v)$. Each 4-path with both endpoints in $N(v)$ has internal vertices in $N^{2}(v)$. Since there are $\left(\begin{array}{l}k \\ 2\end{array}\right)$ such pairs of endpoints and each edge within $N^{2}(v)$ extends to exactly one such path, no edge within $N^{2}(v)$ lies in a triangle with a vertex of $N(v)$. Thus each neighbor of $v$ also lies in no triangle.

We conclude that neighboring vertices both do or both do not lie in triangles. By induction on the distance from $S$, every vertex lies in a triangle. By Lemma 2, each vertex lies in exactly one triangle.

With $A$ being the adjacency matrix of $G$, the matrix $A^{3}$ again counts walks of length 3 . Since each vertex is on one triangle, each diagonal entry is 2 . Since $G$ is $k$-regular, entries for adjacent vertices are $2 k-1$, and by unique $C_{4}$-saturation the remaining entries equal 1 . Hence $A^{3}=J+(2 k-2) A+I$, and again $J$ is expressible as a polynomial in $A$ :

$$
J=A^{3}-(2 k-2) A-I .
$$

Again 1 is an eigenvector of $A$ with eigenvalue $k$ and of $J$ with eigenvalue $n$. All other eigenvalues of $A$ satisfy $p(\lambda)=0$, where

$$
p(\lambda)=\lambda^{3}-(2 k-2) \lambda-1 .
$$

Arguing as in the proof of Theorem $5, p(\lambda)$ cannot be irreducible over $\mathbb{Q}$. If $\lambda$ is rational, then $\lambda= \pm 1$, and $k \in\{1,2\}$. However, $R \neq \varnothing$ requires $k \geq 3$. 
Having shown that $R=\varnothing$, we now consider instances with $N^{2}(S) \neq \varnothing$.

Lemma 8. Let $G$ be a uniquely $C_{4}$-saturated graph with a triangle having vertex set $S$. If $N^{2}(S) \neq \varnothing$, then $G$ is one of the three graphs in Figure 2.

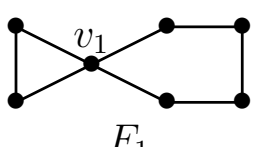

$F_{1}$

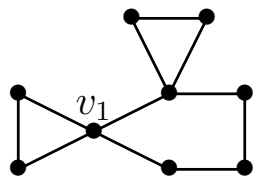

$F_{2}$

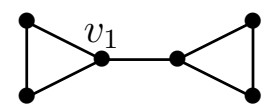

$F_{3}$

Figure 2: Examples having a vertex at distance 2 from a triangle.

Proof. Let $S=\left\{v_{1}, v_{2}, v_{3}\right\}$. In the partition defined in Lemma 6, a 4-path joining $V_{i}^{\prime}$ and $V_{j}^{\prime}$ must pass through $R$. Since $R=\varnothing$, we conclude that only one of $\left\{V_{1}^{\prime}, V_{2}^{\prime}, V_{3}^{\prime}\right\}$ is nonempty; by symmetry, let it be $V_{1}^{\prime}$. Since $G$ has diameter 3 , we have $V_{2}=V_{3}=\varnothing$.

By Lemma 6(f), $V_{1}^{\prime}$ induces a matching. By Lemma 6(b), every vertex of $V_{1}^{\prime}$ thus has degree 2. Consider $w \in V_{1}$ with neighbors $u$ and $v$ in $V_{1}^{\prime}$. If $u$ and $v$ are not adjacent, then a 4-path joining them must use $w$ and the neighbor in $V_{1}^{\prime}$ of one of them. Thus if $w$ has three pairwise nonadjacent neighbors in $V_{1}^{\prime}$, then at least two of them have neighbors in $V_{1}^{\prime}$ that are also neighbors of $w$. This yields two triangles containing $w$, contradicting Lemma 2. We conclude that $w$ cannot have more than three neighbors in $V_{1}^{\prime}$.

If $w \in V_{1}$ has three neighbors in $V_{1}^{\prime}$, then two of them (say $x$ and $y$ ) are adjacent. The only 4-paths that can leave $x$ or $y$ for other vertices of $V_{1}^{\prime}$ end at the remaining neighbor of $w$ or its mate in $V_{1}^{\prime}$. Hence $G=F_{2}$.

If $w \in V_{1}$ has two neighbors in $V_{1}^{\prime}$, then they are adjacent, and no 4-paths can join them to other vertices of $V_{1}^{\prime}$. Hence $G=F_{3}$.

In the remaining case, every vertex of $V_{1}$ has at most one neighbor in $V_{1}^{\prime}$. Since any two vertices of $V_{1}$ are joined by a 4 -path through an edge within $V_{1}^{\prime}$, there can only be two vertices in $V_{1}$, and $G=F_{1}$.

One case remains.

Lemma 9. If $G$ is a uniquely $C_{4}$-saturated graph having a triangle $S$ adjacent to all vertices, then $G$ consists of $S$ and a matching joining $S$ to the remaining (at most three) vertices.

Proof. We have assumed $N^{2}(S)=\varnothing$. Since 4-paths joining vertices in $V_{i}$ must pass through $V_{i}^{\prime}$, each $V_{i}$ has size 0 or 1 . Since $V_{i} \cap V_{j}=\varnothing$ (Lemma $\left.6(\mathrm{a})\right), G$ is as described. 
We can now prove Theorem 1.

Theorem 1. There are exactly ten uniquely $C_{4}$-saturated graphs.

Proof. Trivially, $K_{1}, K_{2}$, and $K_{3}$ are uniquely $C_{4}$-saturated. With girth 5 , there is only $C_{5}$, by Theorem 5 . With girth 3 , Lemma 8 provides three graphs when some vertex has distance 2 from a triangle, and Lemma 9 provides three when there is no such vertex.

\section{References}

[1] P. Erdős, A. Hajnal, and J. W. Moon, A problem in graph theory. Amer. Math. Monthly 71 (1964), 1107-1110.

[2] A. J. Hoffman and R. R. Singleton, On Moore graphs with diameters 2 and 3. IBM J. Res. Develop. 4 (1960), 497-504.

[3] L. T. Ollmann, $K_{2,2}$-saturated graphs with a minimal number of edges. Proc. Third $S E$ Conf. on Combinatorics, Graph Theory, and Computing (Florida Atlantic Univ., Boca Raton, Fla., 1972), 367-392.

[4] O. Pikhurko, Results and open problems on minimum saturated hypergraphs. Ars Combin. 72 (2004), 111-127.

[5] P. Turán, Eine Extremalaufgabe aus der Graphentheorie. Mat. Fiz. Lapok 48 (1941), $436-452$.

[6] P. S. Wenger, Uniquely $C_{k}$-saturated graphs. In preparation.

[7] H. S. Wilf, The friendship theorem. In Combinatorial Mathematics and its Applications (Proc. Conf., Oxford, 1969) (Academic Press, 1971), 307-309. 